\title{
The caudal boundaries of the
}

mesopancreatoduodenum based on the vascular anatomy associated with

pancreaticoduodenectomy

\section{Masahiko Honjo}

Ehime University Graduate School of Medicine

Taiji Tohyama ( $\square$ taitoh1128@gmail.com )

Kurashiki Medical Center

Kohei Ogawa

Ehime University Graduate School of Medicine

Kei Tamura

Ehime University Graduate School of Medicine

Katsunori Sakamoto

Ehime University Graduate School of Medicine

Akihiro Takai

Ehime University Graduate School of Medicine

Jota Watanabe

Ehime Prefectural Central Hospital

Hiromi Ohtani

Ehime Prefectural Central Hospital

\section{Yasutsugu Takada}

Ehime University Graduate School of Medicine

\section{Research Article}

Keywords: Pancreaticoduodenectomy, Mesopancreas, Mesopancreatoduodenum, First jejunal vein, duodenojejunal uncinate process vein

Posted Date: February 8th, 2021

DOl: https://doi.org/10.21203/rs.3.rs-171129/v1

License: (c) (1) This work is licensed under a Creative Commons Attribution 4.0 International License. Read Full License 


\section{Abstract}

\section{Background}

The mesopancreas is important during pancreaticoduodenectomy (PD) in patients with periampullary and pancreatic head carcinoma. This study aimed to investigate whether the duodenojejunal uncinate process vein (DJUV) is a useful anatomical landmark for the caudal border of mesopancreatoduodenum resection.

Methods

This study enrolled 100 adult patients with hepatobiliary pancreatic disease who underwent preoperative multidetector-computed tomography (CT). The anatomy of the key blood vessels involved during PD was analyzed by preoperative CT. The DJUV was defined as the vein draining from the upper jejunum to the superior mesenteric vein adjacent to the uncinate process.

Results

Among 89 cases, the first jejunal vein was the DJUV, whereas the second jejunal vein was the DJUV in 11 cases. The inferior pancreaticoduodenal artery and vein were located on the cranial side of the DJUV in all cases. The distance between the middle colonic artery and the DJUV was within $10 \mathrm{~mm}$ in $81 \%$ of cases, suggesting that resection of the mesopancreatoduodenum cranial to the DJUV provided sufficient dissection of regional lymph nodes around the superior mesenteric artery.

Conclusion

The DJUV may be a useful anatomical landmark for the caudal border of the mesopancreatoduodenum resection during PD.

\section{Background}

Multimodal treatment strategies have gradually improved the prognosis of resectable pancreatic cancer, but it remains poor, with a 5-year survival rate of $20-25 \%$ after surgery [1-7]. Pancreaticoduodenectomy (PD) is the only potential curative treatment and is recognized as one of the most influential determinants of survival $[1,8]$. In 2007, Gockel et al. proposed the concept of the mesopancreas, defined as a firm and well-vascularized structure that extends from the posterior face of the head of the pancreas to behind the superior mesenteric vein (SMV) and artery (SMA). It consists of areolar tissue, adipose tissue, blood vessels, peripheral nerves, and lymph nodes $[9,10]$. This area is recognized as the primary positive resection margin site and the main site of local recurrence [11].

The effectiveness of mesopancreas resection has been reported, but the resection boundaries remain unclear $[12,13]$. The standard procedure for mesopancreas or mesopancreatoduodenum resection has not been established. Anatomical landmarks that can be identified during surgery include anatomical 
membranes and blood vessels. The hepatoduodenal ligament, the common hepatic artery, and the root of the SMA and celiac artery are present on the cranial side of the pancreas, and the anterior renal fascia on the inferior vena cava, renal vein, and aorta are present on the dorsal side [14]. Therefore, the boundaries are relatively clear. However, the caudal borders, particularly the mesenteric dissecting line, are confusing and vary among reports [15-19].

The purpose of the present study was to investigate whether the jejunal vein draining into the SMV at the level of the uncinate process, which we named the "duodenojejunal uncinate process vein (DJUV)", could become a caudal anatomical landmark for mesopancreatoduodenum resection during PD. An anatomical analysis was performed using three-dimensional computed tomography (3D-CT) images based on thinslice CT examinations.

\section{Methods}

\section{Patients}

All procedures involving human participants followed the ethical standards of the institution and research committee and with the 1964 Declaration of Helsinki and its later amendments or comparable ethical standards. This study was approved by the Ethics Review Boards of the Ehime University School of Medicine and Ehime Central Hospital.

A total of 100 patients with hepatobiliary pancreatic disease who underwent preoperative multidetector CT at Ehime University or Ehime Central Hospital between April 2016 and May 2020 were included. The CT data were retrospectively reviewed. There were 58 male and 42 female patients, with a median age of 68 years (range: $37-86$ years).

\section{Definition of DJUV}

The following conditions were required for the caudal border of the mesopancreatoduodenum for radical PD. The excisional area included all blood vessels, the nerve plexus, and regional lymph nodes that should be resected. It should be a macroscopically recognizable anatomical structure during surgery. We have recently focused on the vein that drains from the upper jejunum to the SMV adjacent to the uncinate process via the third and fourth portions of the duodenum. We named this vein the DJUV, and hypothesized that this vein may be a candidate for the caudal border of the mesopancreatoduodenum.

\section{CT protocol and anatomical evaluation}

Multidetector-row CT was performed using a 256-row system, and Synapse Vincent 3D software (Fujifilm Corp., Tokyo, Japan) was used to generate the 3D-CT images. Pancreatic 3D images and arteriographs were obtained during the arterial phase and fused to yield portographs with 0.5 -mm-thick slices. All 3D-CT images were reconstructed by a single surgeon. The tributaries of the SMV were numbered in order from 
the confluence of the splenic vein to distal of the SMV, such as the first and second jejunal veins (J1V and $\mathrm{J} 2 \mathrm{~V}$, respectively), and the jejunal artery branches were numbered from the root of the SMA (Fig. 1).

The following anatomical findings were analyzed: 1) which jejunal vein corresponded to the DJUV; 2) anatomical variations in the inferior pancreaticoduodenal veins (IPDVs) and inferior pancreaticoduodenal artery (IPDA), and their positional relationships with the DJUV were examined; 3 ) the distance between the bifurcation of the middle colonic artery (MCA) and the intersection of the DJUV and SMA (the DJUV intersecting point) was measured, and 4) the number of jejunal arteries branching between the root of the SMA and the DJUV intersecting point was counted.

\section{Results}

\section{Jejunal veins corresponding to the DJUV (Fig. 2)}

Either J1V or J2V corresponded to the DJUV in all cases. J1V was the DJUV (Type 1) in 89 cases, whereas J2V was the DJUV (Type 2) in 11 cases. The DJUV was divided into two subtypes depending on its positional relationship with the SMA, i.e., running dorsal (subtype a) or ventral (subtype b) to the SMA. There were 74 cases of Type 1-a, 15 cases of Type 1-b, 8 cases of Type 2-a, and 3 cases of Type 2-b (Fig. 2). Among the Type 2-a and Type $2 b$ cases, J1V drained into the splenoportal confluence (splenic vein near the confluence of the SMV) in 3 and 2 cases, respectively.

\section{Anatomical variations in the IPDV (Fig. 3)}

As the IPDVs could not be visualized in 8 cases, 92 cases were analyzed. Three variations were identified: the IPDV drained into the DJUV only; it drained into the SMV only, or it drained into both. Variations were observed in 37, 20, and 11 cases, respectively, for Type 1-a patients; in 3, 9, and 1 case for Type 1-b patients; in 1, 6, and 1 case for Type 2-a patients; and in 0, 0, and 3 cases for Type 2-b patients, respectively. In no case did the IPDV join the SMV distal to the DJUV.

\section{Anatomical variations in the IPDA (Fig. 4)}

Only one IPDA was confirmed in most cases. The IPDA branched from the J1A in 53 cases, branched directly from the SMA in 34 cases, branched from the J2A in 3 cases, and branched from the replaced right hepatic artery in 3 cases. Two IPDAs were observed in the remaining 7 cases: one from the J1A and the other from the SMA $(n=4)$; both from the J1A $(n=2)$; and one from the J1A and the other from the J2A $(n=1)$. The IPDA(s) bifurcated from the more cranial side than the MCA and the DJUV intersecting point in all cases.

\section{Distance between the MCA bifurcation and the DJUV intersecting point (Fig. 5)}

According to General Rule for the Study of Pancreas Cancer edited by the Japan Pancreas Society, the lymph nodes located between the bifurcation of the MCA and the root of the SMA are regional in cases of cancer of the head of the pancreas [20]. If the distance between the bifurcation of the MCA and the 
intersection of the DJUV and SMA (DJUV intersecting point) is short, dissecting along the DJUV will reach the SMA at around the bifurcation of the MCA, which is at the distal end of the regional lymph nodes. Matched was defined as a distance of within $10 \mathrm{~mm}$. When the DJUV was equivalent to the J1V (Type 1), the matching rate was $87.8 \%$ for the dorsal type and $73.3 \%$ for the ventral type. When the DJUV was equivalent to the J2V (Type 2), the rates were $50 \%$ and $33.3 \%$, respectively. The matching rate in all cases was $81 \%$. Of the 19 non-matching cases, the DJUV crossed the SMA $11 \mathrm{~mm}$ or more cephalad to the MCA bifurcation in 12 cases.

\section{Number of jejunal arteries branching between the root of the SMA and the DJUV intersecting point}

A single jejunal artery (J1A) was identified in 11 cases. Two arteries (J1A and J2A) were detected in 53 cases, 3 arteries (J1A, J2A, J3A) in 29 cases, and 4 arteries (J1A, J2A, J3A, and J4A, in 7 cases (Table 1).

Table 1

The number of cases in which jejunal arteries branch between the root of the SMA and the DJUV intersecting point

\begin{tabular}{|ll|}
\hline Jejunal artery branch & Number of cases \\
\hline $\mathrm{J} 1 \mathrm{~A}$ & 11 \\
\hline $\mathrm{J} 1 \mathrm{~A}+\mathrm{J} 2 \mathrm{~A}$ & 53 \\
\hline $\mathrm{J} 1 \mathrm{~A}+\mathrm{J} 2 \mathrm{~A}+\mathrm{J} 3 \mathrm{~A}$ & 29 \\
\hline $\mathrm{J} 1 \mathrm{~A}+\mathrm{J} 2 \mathrm{~A}+\mathrm{J} 3 \mathrm{~A}+\mathrm{J} 4 \mathrm{~A}$ & 7 \\
\hline $\begin{array}{l}\text { The English in this document has been checked by at least two professional editors, both native } \\
\text { speakers of English. For a certificate, please see: }\end{array}$ \\
\hline http://www.textcheck.com/certificate/J2zQ9V \\
\hline
\end{tabular}

\section{Surgical techniques for resecting the mesopancreatoduodenum using the DJUV as a landmark}

The following is our standard procedure for typical anatomy.

\section{a) The resection of the caudal border of the mesopancreatoduodenum at the}

\section{DJUV confluence area from the right side}

The SMV is identified in the inferior border of the pancreas. After dividing the gastrocolic trunk, the SMV trunk is detached toward the caudal side. After the uncinate process is identified, the DJUV is confirmed by further detaching to the caudal side of the SMV. The IPDV that connects the uncinate process to the SMV trunk and/or the DJUV should be divided carefully. We sequentially dissect the veins from the region of the SMV-DJUV confluence while inverting the SMV. The DJUV bifurcation is confirmed behind the SMV and serves as a landmark of the endpoint during mesopancreatoduodenum resection. If the IPDV can be resected during the initial stage, the mesopancreatoduodenum resection can easily be performed via the derotation method [16]. 


\section{b) Resection of the caudal border of the mesopancreatoduodenum using the JDUPV}

The retroperitoneum is dissected from the left side of the ligament of Treitz, and the abdominal aorta, inferior vena cava, and left renal vein are exposed. After dividing the jejunum $20-30 \mathrm{~cm}$ from the anal side of the ligament of Treitz, the marginal vessels are followed to the DJUV. As shown in the present study, the DJUV runs with the jejunal artery dorsally via the dorsal side of the SMA in most cases.

Therefore, the DJUV is easily identified by dividing the mesojejunum serosa from the dorsal side. The SMA is surrounded by a nerve plexus and was identified at its left-anterior aspect; the MCA was identified around the intersecting point of the DJUV. Then, the dissection of the mesojejunum from the ventral side communicated with the dissection from the dorsal side with the DJUV as a caudal surgical margin. The dissection of the mesojejunum was promoted to the cranial side toward the origin of the SMA along with the regional lymph node resection, and the common trunk of the IPDA/J1A was divided at the posterior aspect of the SMA. The IMV was dissected from the mesojejunum as the left side surgical margin. The dissection of the anterior-to-posterior aspect of the SMA was promoted to its origin, and the ligament of Treitz was dissected at the left aspect of the SMA. After dividing the ligament of Treitz, the left renal vein was identified by prior dissection as the dorsal surgical margin. The dissection of the mesopancreatoduodenum and uncinate process from the SMA was almost completed toward the right aspect of the SMA with rotation of the SMA counterclockwise using a left-sided approach. The dissected part of the mesopancreatoduodenum on the left side is connected to the exfoliated area around the DJUP confluence of the SMV; excision of the caudal side of the mesopancreatoduodenum is then completed. Finally, the jejunum and mesojejunum are pulled through to the patient's right side, and the connective tissue between the SMA and the head of the pancreas, which is called the "second portion of the pancreatic head plexus (PLphII)" [20] in Japan, was dissected toward the origin of the SMA.

\section{Discussion}

In 1982, Heald et al. described the concept of the mesorectum, the importance of the surgical principle of the "holy plane", and total mesorectal excision during rectal cancer surgery [21]. In 2007, inspired by the concept of the mesorectum, Gockel et al. advocated the concept of the mesopancreas [9]. In Japan, the "pancreatic head nerve plexus II" was defined in General Rule for the Study of Pancreas Cancer [20]. Kawabata et al. advocated the "mesopancreatoduodenum" as an advance of the mesopancreas concept, which expanded the area for lymphadenectomy to the left side of the SMA $[13,15]$. Although several surgical procedures for mesopancreas resection have been proposed, the caudal borders, particularly the jejunal mesenteric dissecting line, vary in each report. As examples, the landmarks that have been reported are the J1A [15], the line between the J1A and J2A [16], the J2A and J2V [18], and the J1V with combined resection of the confluence of the jejunal veins [19]. The present study is the first to propose the concept of mesopancreatoduodenum resection guided by the DJUV, which is the jejunal mesenteric vein connected to the caudal side of the uncinate process. 
Several recent reports have implied the importance of jejunal vein anatomy for PD [17, 18, 22-24]. In some studies [18, 23], the jejunal veins were named from the peripheral drainage territories of the respective jejunal arteries, i.e., the first jejunal vein is the vein that drains the J1A territory. In contrast, we and others [17] defined the first or second tributaries of the SMV as J1V or J2V based on the relationship with the uncinate process. Therefore, "the first jejunal trunk (FJT) [18]" or "the first jejunal venous trunk (JVT) [23]", which drained the first and second jejunal veins in previous studies, corresponds to the J1V in the latter studies, including ours. In the present study, the J1V was regarded as the DJUV (Type 1) in 89\% of cases, in which the vein ran on the dorsal side of the SMA in 74 cases (83\%). Previous studies have reported that the J1V runs dorsal to the SMA in $63-91 \%$ of cases [17, 18, 23-25]. In the other 11 cases $(11 \%)$, the J2V was regarded as the DJUV. Nagakawa et al. reported that the J2V was connected to the uncinate process in 10 of 41 cases (23.8\%) [22].

Few studies have analyzed the anatomical relationships between the jejunal veins and the IPDA and the IPDV, which are included in the mesopancreatoduodenum [18]. In the present results, three IPDV drainage patterns were observed (Fig. 3). In all cases, the IPDVs joined the DJUV or the more cranial side of the SMV. This suggests that the SMV-DJUV confluence and IPDVs can be easily confirmed while rotating the SMV when dissecting along the DJUV, and it is possible to safely dissect between the uncinate process and the SMV.

As shown in Fig. 4, there were several variations in the branching pattern of the IPDA. The IPDA arose from J1A or J2A in most cases, and all branches were located on the cranial side of the DJUV. This means that all of the IPDA(s) can be easily identified and divided by dissecting the area around the SMA starting from the intersecting point of the DJUV on the cranial side, all the IPDA(s) can be easily identified and divided. This is effective in terms of the "artery-first approach" [26], especially in cases of the DJUV running on the dorsal side of the SMA.

In Japan, the lymph nodes located between the bifurcation of the MCA and the root of the SMA are considered regional lymph nodes, and lymphadenectomy in this area around the SMA is required during standard PD for cancer of the pancreatic head. The frequency of lymph node metastasis in that area has been reported to be 11-40\% [27-31]. Nakamura et al. reported that the median distance of the "FJV (first jejunal vein)" was $0 \mathrm{~mm}$ from the MCA in the CT images of 54 patients [17]. In the present study using 3D-CT images, the positional relationship between the DJUV and the bifurcation of the MCA matched (within $10 \mathrm{~mm}$ ) in $81 \%$ of cases. The matching rate was $88 \%$ for the most frequent type (Type $1-a)$. These results indicate that the dissection along the DJUV will reach close to the vicinity of the root of the MCA in most cases, and subsequent dissection around the SMA toward the cranial direction facilitates complete resection of these regional lymph nodes. However, the matching rate was lower in Type 2 than in Type 1 cases (50\% in Type 2-a and 33.3\% in Type 2-b). In addition, the DJUV was located $11 \mathrm{~mm}$ or more cranial from the MCA in $12 \%$ of the cases. Attention must be given to these cases from the perspective of regional lymphadenectomy. 
If pancreatic cancer has invaded the confluence of the DJUVs, the confluence must be resected to achieve no residual tumor (i.e., R0). Pancreaticoduodenectomy with DJUV resection is feasible for most pancreatic ductal adenocarcinomas $[19,32,33]$. However, Kobayashi et al. reported that $87.8 \%$ of DJUVs have multiple jejunal vein branches, and diameter is significantly associated with the number of jejunal vein branches. One of 32 with a DJUV sacrificed showed severe congestion of the jejunal limb requiring an emergency jejunal resection [23]. In this study, the DJUV (J1V) had one to five jejunal veins entering the DJUV in most typical Type 1a cases, and J1V and J2V were nearly confluent in 37 of 89 cases of Type 1a (41.6\%) (data are not shown). If pancreatic cancer has invaded the DJUV in cases where the DJUV corresponds to $\mathrm{J} 2$ and cases in which $\mathrm{J} 1 \mathrm{~V}$ and $\mathrm{J} 2 \mathrm{~V}$ are nearly confluent to the SMV, it is necessary to consider reconstruction based on the anatomical findings, including peripheral arcades. If the tumor has invaded the DJUV or SMV, we generally resect the confluence of the DJUV and SMV, but the cut line of the jejunal mesentery is the same in the peripheral region of the DJUV.

Although these anatomical advantages suggest the utility of the DJUV as a landmark for mesopancreatoduodenum resection, there are some drawbacks regarding the jejunal arteries. In the present study, only the J1A or J1A + J2A originated from the SMA between its root and the DJUV intersecting point in $64 \%$ of cases. However, in the other $36 \%$ of cases, the J3A or J3A $+J 4 A$ branched between the two points. Ishikawa et al. reported cases with three or more jejunal arteries branching from the SMA above the intersection of the FJT, corresponding to the J1V in the present study [18]. In such cases, if the jejunum and mesenteric division are transected along the DJUV, multiple jejunal arteries, such as J3A and J4A, must be transected, which may cause ischemic damage to the elevated jejunum. In clinical practice, circulation from the arterial arcade occasionally compensates for the blood supply, but this is not evaluated preoperatively. Therefore, it may be necessary to shift the cut line of the mesojejunum to the more cranial side (i.e., along J2A).

The present study had some limitations. One is the retrospective design, which caused bias. Another is the relatively small sample size. Because the vessel anatomy around the head of the pancreas varies widely, further investigation of a larger number of cases is needed to assess the anatomical advantages of the DJUV for mesopancreatoduodenum resection. Furthermore, surgical outcomes and patient prognosis should be evaluated to confirm the validity of the DJUV-guided method.

\section{Conclusions}

Based on an analysis of the vascular anatomy and positional relationships between the DJUV and key vessels, the present study suggests the accuracy of the DJUV as a landmark for mesopancreatoduodenum resection. Dissecting along the DJUV as a caudal border may be useful for safe and oncologically curative PD.

\section{Abbreviations}


PD: pancreaticoduodenectomy; SMV: superior mesenteric vein; SMA: superior mesenteric artery; CT: computed tomography; FJV: first jejunal vein; DJUV: duodenojejunal uncinate process vein; JV: jejunal vein; IPDV: inferior pancreaticoduodenal vein; MCA: middle colic artery; 3D: three-dimensional; J1V : first jejunal vein; J2V: second jejunal vein; IPDA: inferior pancreaticoduodenal artery; PLphll : pancreatic head plexus II

\section{Declarations}

\section{Acknowledgments}

The authors thank A.O., S. Inc. for illustrating the figures.

\section{Authors' contributions}

TT and MH contributed to the study conception and design. TT, MH, KT, AT, KS, KO, JW, HO, and YT participated data collection. TT, MH, and YT contributed the analysis and interpretation of the data. TT and $\mathrm{MH}$ drafted the manuscript. TT and YT critical reviewed. All authors read and approved the final manuscript.

\section{The availability of data and materials}

The datasets used and/or analyzed during the current study are available from the corresponding author on reasonable request.

\section{Funding}

Not applicable.

\section{Ethical approval and consent to participate}

All procedures performed in studies involving human participants were in accordance with the ethical standards of the institution and research committee and with the 1964 Helsinki declaration and its later amendments or comparable ethical standards. This study was approved by the Ethics Review Boards of the Ehime University School of Medicine and Ehime Central Hospital. Informed consent was by the optout principle and was disclosed on the study website (https://www.m.ehime-u.ac.jp/school/surgery1/wpcontent/uploads/2020/10/4bd601621bf36c0ac5416707937c91d1.pdf), which included general information and provided an opportunity to decline to participate in this study.

\section{Consent for publication}

Not applicable.

\section{Competing interests}

The authors declare that they have no conflicts of interest. 


\section{References}

1. Hartwig W, Hackert T, Hinz U, Gluth A, Bergmann F, Strobel O, et al. (2011) Pancreatic cancer surgery in the new millennium: better prediction of outcome. Ann Surg 254:311-319.

2. Oettle H, Neuhaus P, Hochhaus A, Hartmann JT, Gellert K, Ridwelski K, et al. (2013) Adjuvant chemotherapy with gemcitabine and long-term outcomes among patients with resected pancreatic cancer: the CONKO-001 randomized trial. Jama 310:1473-1481.

3. Cameron JL, He J. (2015) Two thousand consecutive pancreaticoduodenectomies. J Am Coll Surg 220:530-536.

4. Uesaka K, Boku N, Fukutomi A, Okamura Y, Konishi M, Matsumoto I, et al. (2016) Adjuvant chemotherapy of S-1 versus gemcitabine for resected pancreatic cancer: a phase 3, open-label, randomised, non-inferiority trial (JASPAC 01). Lancet 388:248-257.

5. Conroy T, Hammel P, Hebbar M, Ben Abdelghani M, Wei AC, Raoul JL, et al. (2018) FOLFIRINOX or Gemcitabine as Adjuvant Therapy for Pancreatic Cancer. N Engl J Med 379:2395-2406.

6. Motoi F, Kosuge T, Ueno H, Yamaue H, Satoi S, Sho M, et al. (2019) Randomized phase II/III trial of neoadjuvant chemotherapy with gemcitabine and S-1 versus upfront surgery for resectable pancreatic cancer (Prep-02/JSAP05). Jpn J Clin Oncol 49:190-194.

7. Motoi F, Unno M. (2020) Adjuvant and neoadjuvant treatment for pancreatic adenocarcinoma. Jpn J Clin Oncol 50:483-489.

8. Maeda S, Moore AM, Yohanathan L, Hata T, Truty MJ, Smoot RL, et al. (2020) Impact of resection margin status on survival in pancreatic cancer patients after neoadjuvant treatment and pancreatoduodenectomy. Surgery 167:803-811.

9. Gockel I, Domeyer M, Wolloscheck T, Konerding MA, Junginger T. (2007) Resection of the mesopancreas (RMP): a new surgical classification of a known anatomical space. World J Surg Oncol 5:44.

10. Quero G, Fiorillo C, Menghi R, Cina C, Galiandro F, Longo F, et al. (2020) Total mesopancreas excision for periampullary malignancy: a single-center propensity score-matched comparison of long-term outcomes. Langenbecks Arch Surg 405:303-312.

11. Gaedcke J, Gunawan B, Grade M, Szöke R, Liersch T, Becker H, et al. (2010) The mesopancreas is the primary site for $\mathrm{R} 1$ resection in pancreatic head cancer: relevance for clinical trials. Langenbecks Arch Surg 395:451-458.

12. Ramia JM, De-la-Plaza R, Manuel-Vazquez A, Lopez-Marcano A, Morales R. (2018) Systematic review of the mesopancreas: concept and clinical implications. Clin Trans/ Oncol 20:1385-1391.

13. Nappo G, Perinel J, El Bechwaty M, Adham M. (2016) The Standardization of Pancreatoduodenectomy: Where Are We? Pancreas 45:493-502.

14. Kitagawa H, Tajima H, Nakagawara H, Makino I, Miyashita T, Terakawa H, et al. (2014) A modification of radical antegrade modular pancreatosplenectomy for adenocarcinoma of the left 
pancreas: significance of en bloc resection including the anterior renal fascia. World $J$ Surg 38:2448-2454.

15. Kawabata Y, Tanaka T, Nishi T, Monma H, Yano S, Tajima Y. (2012) Appraisal of a total mesopancreatoduodenum excision with pancreaticoduodenectomy for pancreatic head carcinoma. Eur $J$ Surg Oncol 38:574-579.

16. Sugiyama M, Suzuki Y, Nakazato T, Yokoyama M, Kogure M, Abe N, et al. (2016) Intestinal derotation procedure for facilitating pancreatoduodenectomy. Surgery 159:1325-1332.

17. Nakamura M, Nakashima H, Tsutsumi K, Matsumoto H, Muta Y, Ueno D, et al. (2013) First jejunal vein oriented mesenteric excision for pancreatoduodenectomy. J Gastroenterol 48:989-995.

18. Ishikawa Y, Ban D, Matsumura S, Mitsunori Y, Ochiai T, Kudo A, et al. (2017) Surgical pitfalls of jejunal vein anatomy in pancreaticoduodenectomy. J Hepatobiliary Pancreat Sci 24:394-400.

19. Inoue Y, Saiura A, Yoshioka R, Ono Y, Takahashi M, Arita J, et al. (2015) Pancreatoduodenectomy With Systematic Mesopancreas Dissection Using a Supracolic Anterior Artery-first Approach. Ann Surg 262:1092-1101.

20. Society JP. Classification of Pancreatic Carcinoma. 4th English Edition ed, Tokyo: Kanehara \& Co. Ltd.; 2017.

21. Heald RJ, Husband EM, Ryall RD. (1982) The mesorectum in rectal cancer surgery-the clue to pelvic recurrence? Br J Surg 69:613-616.

22. Nagakawa Y, Hosokawa Y, Sahara Y, Takishita C, Hijikata Y, Osakabe H, et al. (2018) Approaching the superior mesenteric artery from the right side using the proximal-dorsal jejunal vein preisolation method during laparoscopic pancreaticoduodenectomy. Surg Endosc 32:4044-4051.

23. Kobayashi Y, Sakamoto Y, Arita J, Akamatsu N, Kaneko J, Hasegawa K, et al. (2018) Vascular anatomy of the jejunal mesentery and complications associated with division of the first jejunal venous trunk during pancreaticoduodenectomy. J Surg Oncol 117:1297-1304.

24. Sugiyama M, Suzuki Y, Nakazato T, Yokoyama M, Kogure M, Matsuki R, et al. (2020) Vascular Anatomy of Mesopancreas in Pancreatoduodenectomy Using an Intestinal Derotation Procedure. World J Surg. https://doi.org/10.1007/s00268-020-05605-z.

25. Negoi I, Beuran M, Hostiuc S, Negoi RI, Inoue Y. (2018) Surgical Anatomy of the Superior Mesenteric Vessels Related to Pancreaticoduodenectomy: a Systematic Review and Meta-Analysis. $J$ Gastrointest Surg 22:802-817.

26. Sanjay P, Takaori K, Govil S, Shrikhande SV, Windsor JA. (2012) 'Artery-first' approaches to pancreatoduodenectomy. Br J Surg 99:1027-1035.

27. Matsuno S, Egawa S, Fukuyama S, Motoi F, Sunamura M, Isaji S, et al. (2004) Pancreatic Cancer Registry in Japan: 20 years of experience. Pancreas 28:219-230.

28. Kanda M, Fujii T, Nagai S, Kodera Y, Kanzaki A, Sahin TT, et al. (2011) Pattern of lymph node metastasis spread in pancreatic cancer. Pancreas 40:951-955. 
29. Kawabata Y, Tanaka T, Ishikawa N, Hayashi H, Tajima Y. (2016) Modified total mesopancreatoduodenum excision with pancreaticoduodenectomy as a mesopancreatic plane surgery in borderline resectable pancreatic cancer. Eur J Surg Oncol 42:698-705.

30. Qian L, Xie J, Xu Z, Deng X, Chen H, Peng C, et al. (2020) The Necessity of Dissection of No. 14 Lymph Nodes to Patients With Pancreatic Ductal Adenocarcinoma Based on the Embryonic Development of the Head of the Pancreas. Front Onco/ 10:1343.

31. Imamura T, Yamamoto Y, Sugiura T, Okamura Y, Ito T, Ashida R, et al. (2020) Reconsidering the Optimal Regional Lymph Node Station According to Tumor Location for Pancreatic Cancer. Ann Surg Oncol. https://doi.org/10.1245/s10434-020-09066-5.

32. Hosokawa Y, Nagakawa Y, Sahara Y, Takishita C, Nakajima T, Hijikata Y, Osakabe H, Shirota T, Saito K, Yamaguchi H, Inoue K, Katsumata K, Tsuchiya T, Sofuni A, Itoi T, Tsuchida A (2018) Surgical Outcomes of Pancreaticoduodenectomy for Pancreatic Cancer with Proximal Dorsal Jejunal Vein Involvement. J Gastrointest Surg 22(7):1179-1185. https://doi.org/10.1007/s11605-018-3722-0

33. Honda M, Nagakawa Y, Akashi M, Hosokawa Y, Osakabe H, Takishita C, Nishino H, Tsuchida A (2020) Clinical impact of pancreaticoduodenectomy for pancreatic cancer with resection of the secondary or later branches of the superior mesenteric vein. J Hepatobiliary Pancreat Sci. https://doi.org/10.1002/jhbp.789

\section{Figures}

Fig 1. Typical images of the duodenojejunal uncinate process vein (DJUV).
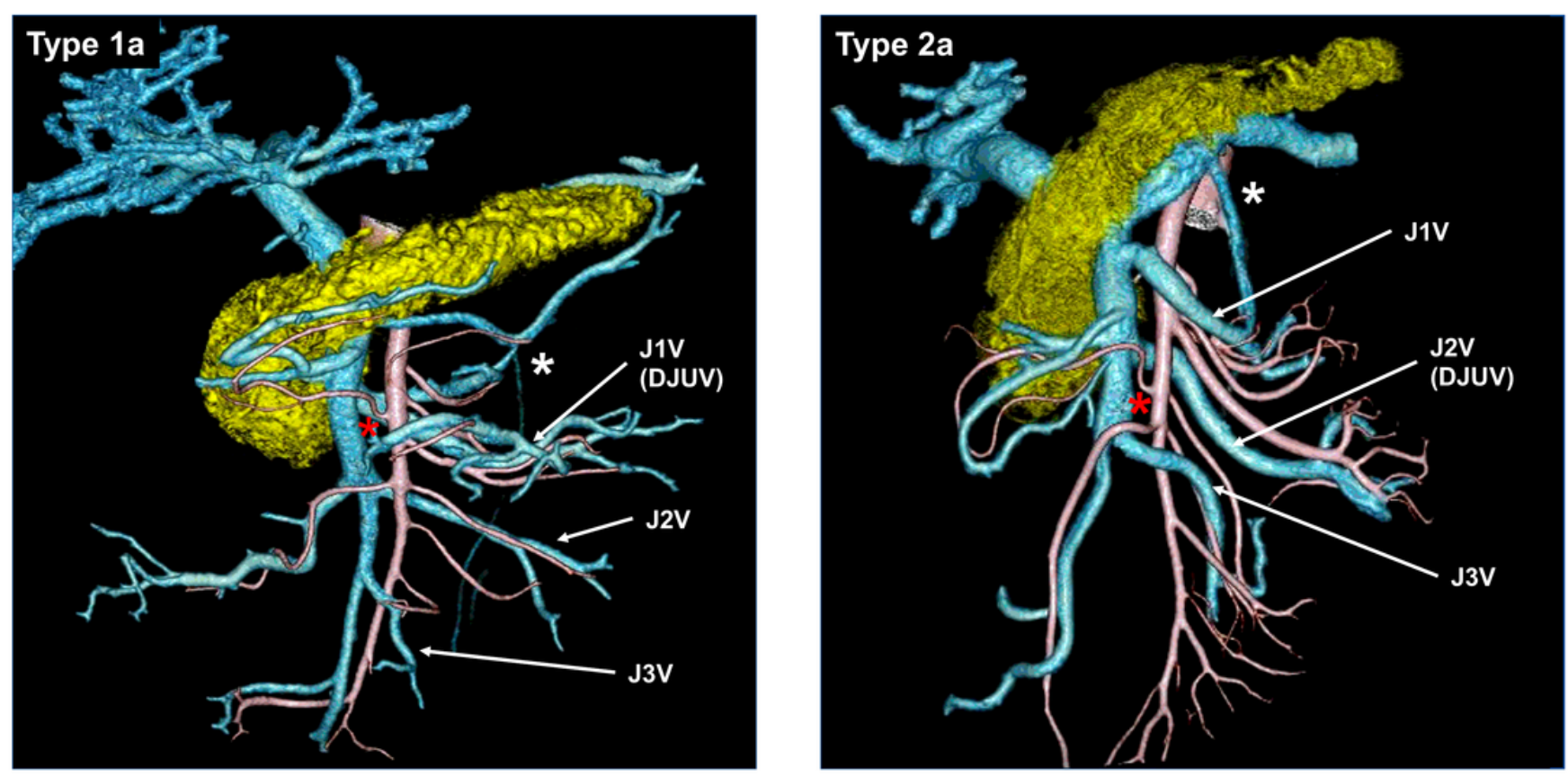

Figure 1 
Typical DJUV images a) Type 1-a, in which the J1V corresponds to the DJUV, runs dorsal to the SMA. b) Type 2-a, in which the J2V corresponds to the DJUV, runs dorsal to the SMA. DJUV, duodenojejunal uncinate process vein; J1-4A, first to fourth jejunal arteries; J1V-J3V, first to third jejunal veins; white asterisk, inferior mesenteric vein; red asterisk, middle colic artery

Fig. 2 Anatomical variations of the duodenojejunal uncinate process vein (DJUV).

\begin{tabular}{|c|c|c|c|c|c|}
\hline DJUV & J1V & & J2V & & Total \\
\hline & Type 1a & Type 2a (V1 vent.) & Type 2a (V1 dor.) & Type 2a (V1 spl.) & \\
\hline \multirow[t]{2}{*}{ Dorsal } & & & & & $n=82$ \\
\hline & Type $1 \mathrm{~b}$ & Type 2b (V1 vent.) & & Type 2b (V1 spl.) & \\
\hline \multirow[t]{2}{*}{ Ventral } & & & & & $\mathrm{n}=18$ \\
\hline & & & & & \\
\hline Total & $\mathrm{n}=89$ & & $\mathrm{n}=11$ & & \\
\hline
\end{tabular}

Figure 2

Anatomical variations in the duodenojejunal uncinate process vein (DJUV) The J1V was the DJUV (Type 1) in 89 cases, whereas the J2V was the DJUV in 11 cases (Type 2). Next, the DJUV was divided into two subtypes depending on its positional relationship with the SMA, i.e., running dorsal (subtype a) or ventral (subtype b) to the SMA. There were 74 cases of Type 1-a, 15 cases of Type 1-b, 8 cases of Type 2-a, and 3 cases of Type 2-b. The J1V ran along the ventral side of the SMA in 2 cases of Type 2-a, along the dorsal side of the SMA in 3 cases, and the J1V drained into the splenic vein in 3 cases. In Type 2-b, the J1V ran along the ventral side of the SMA in 1 case, and the J1V drained into the splenic vein in 2 cases. DJUV, duodenojejunal uncinate process vein; J1V first jejunal vein; J2V second jejunal vein; V1 vent., the first jejunal vein running ventral to the superior mesenteric artery; V1 dor., the first jejunal vein running dorsally to the superior mesenteric artery; V1 spl., first jejunal vein drained into the splenoportal confluence 
Fig.3 Anatomical variations of the inferior pancreaticoduodenal vein

1) Type 1a $n=74$ (Unknown 6)

DJUV

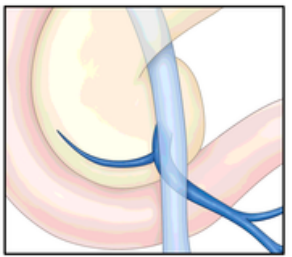

$\mathrm{n}=37$
SMV

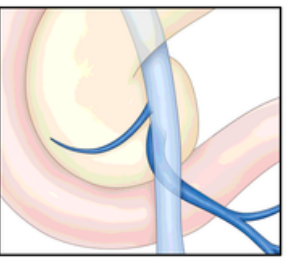

$\mathrm{n}=20$
DJUV +SMV

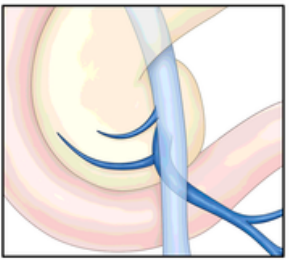

$n=11$

2) Type 1b $n=15$ (Unknown 2)

DJUV

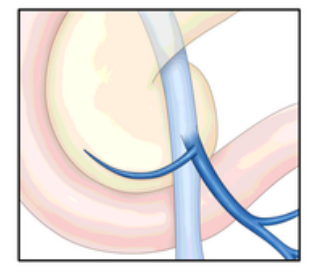

$n=3$
SMV

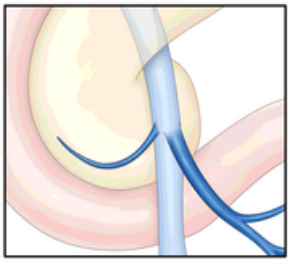

$\mathrm{n}=9$

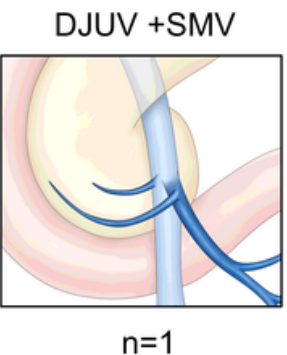

3) Type $2 a \quad n=8$

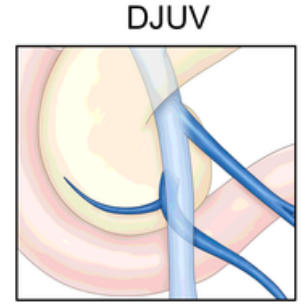

$\mathrm{n}=1$

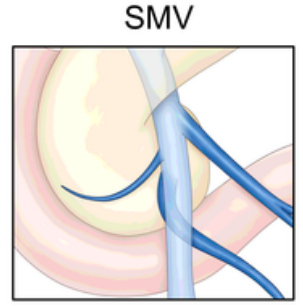

$n=6$
DJUV +SMV

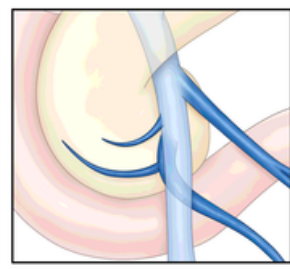

$n=1$

4) Type $2 b \quad n=3$

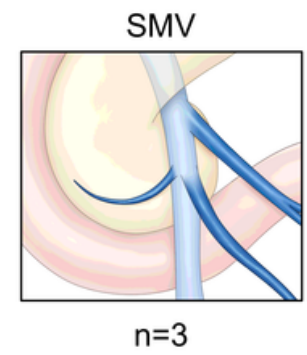

\section{Figure 3}

Anatomical variations in the inferior pancreaticoduodenal vein Three variations were identified: blood return to the DJUV only; blood return to the SMV only; and blood return to both. 1) Type 1-a included 37, 20, and 11, respectively. 2) Type 1-b included 3, 9, and 1, respectively. 3) Type 2-a included 1, 6, and 1, respectively. 4) Type 2-b included 0, 0, and 3, respectively. DJUV, duodenojejunal uncinate process vein; SMV, superior mesenteric vein 
Fig.4 Anatomical variations of the inferior pancreaticoduodenal artery
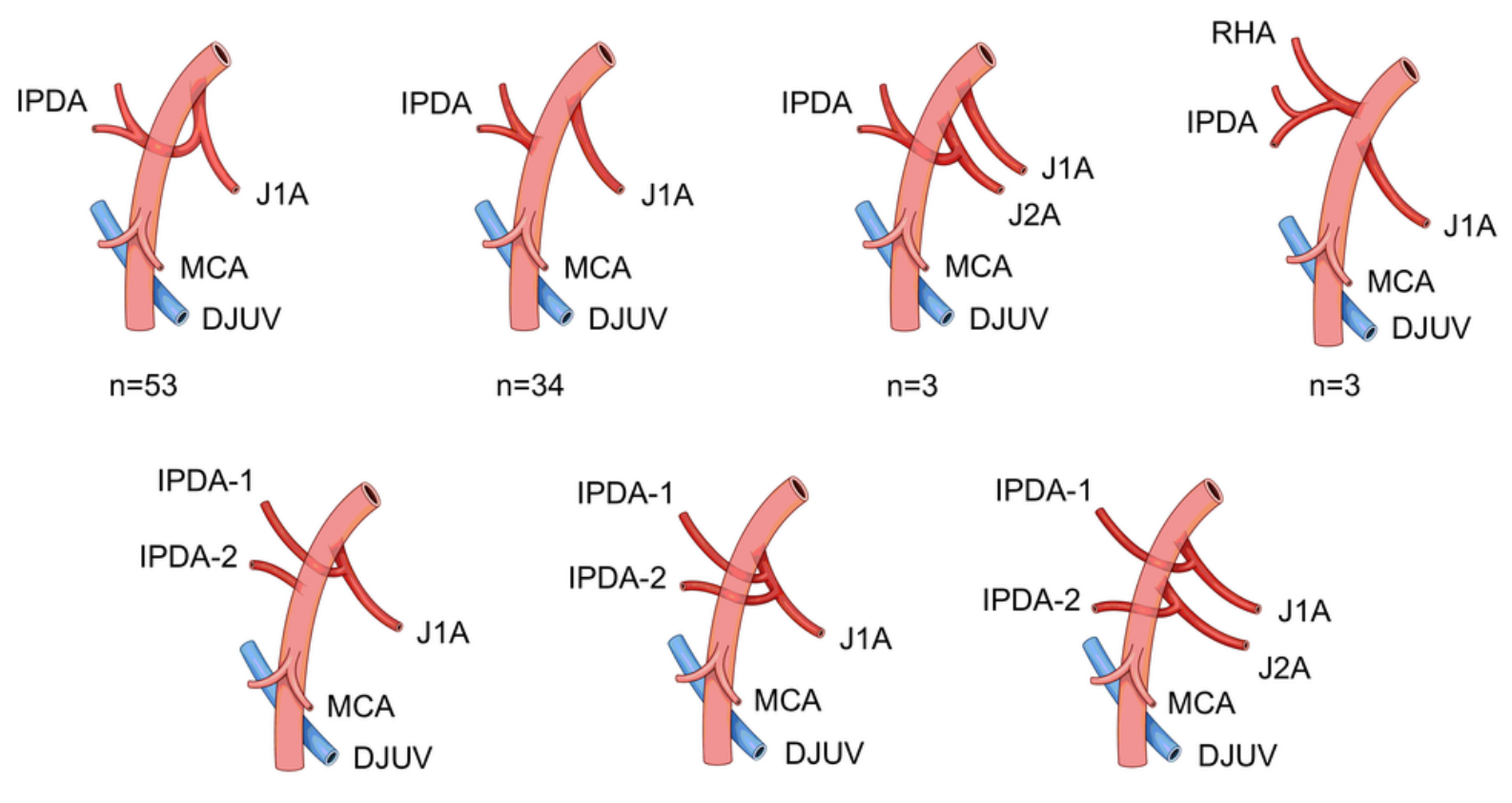

$\mathrm{n}=4$

$\mathrm{n}=2$

$\mathrm{n}=1$

\section{Figure 4}

Anatomical variations in the inferior pancreaticoduodenal artery A single IPDA branched from the J1A in 53 cases, branched directly from the SMA in 33 cases, branched from the J2A in 3 cases, and branched from a replaced right hepatic artery in 3 cases. Two IPDAs were observed in the remaining seven cases: one from the J1A and the other from the SMA ( $n=4)$; both from the J1A $(n=2)$; and one from the J1A and the other from the J2A ( $n=1)$. IPDA, inferior pancreatoduodenal artery; J1A, first jejunal artery; J2A, second jejunal artery; RHA, right hepatic artery; MCA, middle colic artery; DJUV, duodenojejunal uncinate process vein 
Fig. 5 Positional relationship of the middle colic artery and the duodenojejunal uncinate process vein (DJUV)

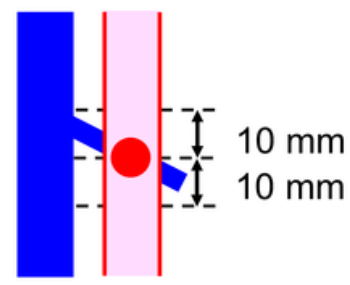

Match rate (within $10 \mathrm{~mm}$ from the MCA): $81 \%$

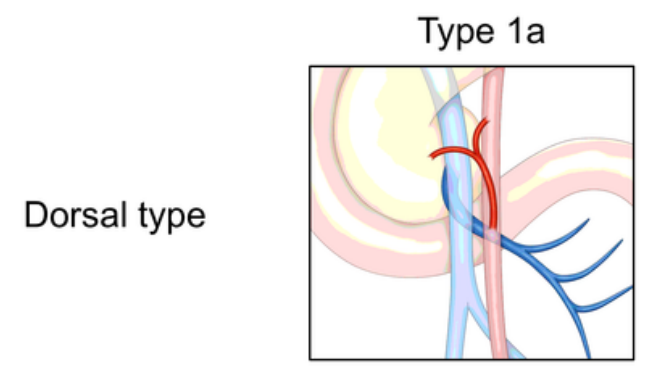

$65 / 74(87.8 \%)$

Type $1 b$

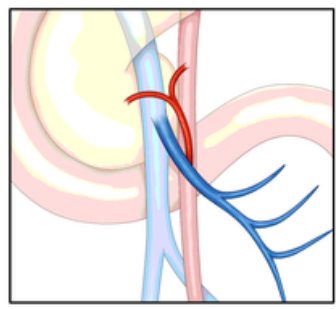

$11 / 15(73.3 \%)$
Type 2a

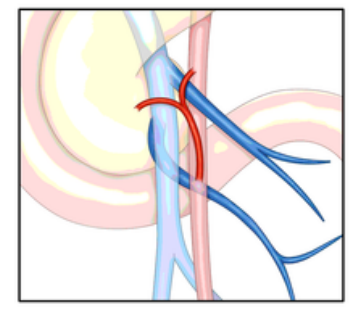

$4 / 8(50.0 \%)$

Type $2 b$

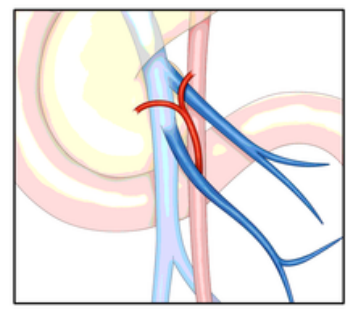

$1 / 3(33.3 \%)$

\section{Figure 5}

Positional relationship of the middle colic artery and the duodenojejunal uncinate process vein (DJUV) Matching was defined as the distance between the bifurcation of the MCA and the intersection of the DJUV and SMA (DJUV crossing point) within $10 \mathrm{~mm}$. Type 1-a had a match rate of 87.8\%. Type 1-b had a match rate of $73.3 \%$. The match rates of Type 2-a and Type 2-b were lower, at 50\% and 33.3\%, respectively. The match rate of all cases was $81 \%$. DJUV, duodenojejunal uncinate process vein; MCA, middle colic artery 